\title{
CALENDAR/MEETING NEWS
}

\section{MEDICAL IMAGING 1995 (SPIE)}

San Diego, CA

February 26-March 2, 1995

For further information, contact: Matthew Krough, SPIE, P.O. Box 10, Bellingham, WA 98225. Telephone 206-676-3290. Fax 206-647-1445.

\author{
CAR 95-9TH COMPUTER-ASSISTED \\ RADIOLOGY INTERNATIONAL SYMPOSIUM \\ includes \\ ISPRAD VII \\ and \\ EuroPACS 1995 \\ Berlin, Germany \\ June 21-24, 1995
}

For further information, contact: Heinz U. Lemke, Technische Universitat Berlin. Institut fur Technische Informatik, Sekr. CG FR 3-3. Helga Kallan, Franklinstr. 28-29, D-10587 Berlin, Germany. Telephone 49-(0)30-314-73100. Fax 49-(0)30-314-21103. 